\title{
ZnO dense nanowire array on a film structure in a single crystal domain texture for optical and photoelectrochemical applications
}

\author{
Miao Zhong ${ }^{1}$, Yukio Sato ${ }^{2}$, Mario Kurniawan $^{1}$, Aleksandra Apostoluk ${ }^{3}$, \\ Bruno Masenelli ${ }^{3}$, Etsuo Maeda ${ }^{1}$, Yuichi Ikuhara ${ }^{2}$ and \\ Jean-Jacques Delaunay ${ }^{1}$ \\ ${ }^{1}$ School of Engineering, The University of Tokyo, 7-3-1 Hongo, Bunkyo-ku, Tokyo 113-8656, Japan \\ 2 Institute of Engineering Innovation, School of Engineering, The University of Tokyo, 2-11-16 Yayoi, \\ Bunkyo-Ku, Tokyo 113-8656, Japan \\ ${ }^{3}$ Institut des Nanotechnologies de Lyon (INL, CNRS UMR-5270), INSA Lyon, Université de Lyon, \\ 7 Avenue Jean Capelle, Villeurbanne F-69621, France
}

E-mail: zhong.miao@scale.t.u-tokyo.ac.jp and jean@mech.t.u-tokyo.ac.jp

\begin{abstract}
A single crystal domain texture quality (a unique in-plane and out-of-plane crystalline orientation over a large area) $\mathrm{ZnO}$ nanostructure of a dense nanowire array on a thick film has been homogeneously synthesized on a-plane sapphire substrates over large areas through a one-step chemical vapor deposition (CVD) process. The growth mechanism is clarified: a single crystal $\left[0^{-} 21\right]$ oriented $\mathrm{ZnAl} 2 \mathrm{O} 4$ buffer layer was formed at the $\mathrm{ZnO}$ film and the a-plane sapphire substrate interface via a diffusion reaction process during the CVD process, providing improved epitaxial conditions that enable the synthesis of the high crystalline quality $\mathrm{ZnO}$ nanowire array on a film structure. The high optoelectronic quality of the $\mathrm{ZnO}$ nanowire array on a film sample is evidenced by the free exitonic emissions in the low-temperature photoluminescence spectroscopy. A carrier density of $\sim 1017 \mathrm{~cm}-3$ with an n-type conductivity of the $\mathrm{ZnO}$ nanowire array on a film sample is obtained by electrochemical impedance analysis. Finally, the $\mathrm{ZnO}$ nanowire array on a film sample is demonstrated to be an ideal template for a further synthesis of a single crystal quality $\mathrm{ZnO}-\mathrm{ZnGa} 2 \mathrm{O} 4$ core-shell nanowire array on a film structure. The fabricated $\mathrm{ZnO}-\mathrm{ZnGa} 2 \mathrm{O} 4$ sample revealed an enhanced anticorrosive ability and photoelectrochemical performance when used as a photoanode in a photoelectrochemical water splitting application.
\end{abstract}

\section{Introduction}

Due to their unique optical, electrical and semiconducting properties, one-dimensional $\mathrm{ZnO}$ nanowire $(\mathrm{NW})$ arrays have been extensively studied in the past decade for their potential use in electronic, photonic, electromechanical and electrochemical applications [1-6]. As $\mathrm{ZnO}$ is an abundant and cheap material, recent scientific studies focused on an efficient fabrication and assembly of high-quality $\mathrm{ZnO} \mathrm{NW}$ arrays by simple and easy controllable methods which can be commercially competitive. Compared to some sophisticated technologies such as molecular beam epitaxy (MBE), atomic layer deposition (ALD) and electron beam lithography, chemical vapor deposition (CVD) and wet chemical growth 
are substantially cost-effective methods [7-9], and, therefore, desirable for mass production of the $\mathrm{ZnO} \mathrm{NW}$ arrays. However, multiple challenges still remain to be addressed, as far as these simple fabrication methods are concerned. One of the most difficult issues is the realization of a high texture quality of a $\mathrm{ZnO} \mathrm{NW}$ array (i.e., a single crystal quality and a single crystal domain orientation over a large area) by a CVD or wet chemical process. A good texture quality is essential for the development of high-performance photonic, electronic and optoelectronic devices [10-12]. For example, a large decrease in the electron mobility from 120 to $30 \mathrm{~cm}^{2} \mathrm{~V}^{-1} \mathrm{~s}^{-1}$ is obtained between a $\mathrm{ZnO}$ thin film with a unique crystalline domain and a $\mathrm{ZnO}$ thin film having two domains with distinct in-plane orientations [10]. This is explained as the result of the rotational defects in $\mathrm{ZnO}$ [10]. The decreased electron mobility may affect the charge collection efficiency, thus a high texture quality of the $\mathrm{ZnO} \mathrm{NW}$ arrays is crucial to obtain enhanced performances in electronic, optoelectronic and piezoelectric devices.

Generally, epitaxial growth is a pre-requisite to achieve a high texture quality of the fabricated materials [10, 13-15]. $\mathrm{GaN}$ matches the in-plane geometry and lattice constants of $\mathrm{ZnO}$, and therefore has been used as an epitaxial substrate for the growth of the $\mathrm{ZnO} \mathrm{NW}$ arrays, regardless of the high cost of GaN single crystals. However, the unintentional doping of the $\mathrm{ZnO} \mathrm{NW}$ array with $\mathrm{Ga}$ and/or $\mathrm{N}$ easily occurs during the growth processes [16], and this unintentional doping renders the electronic properties of the fabricated $\mathrm{ZnO} \mathrm{NWs}$ difficult to control. On the other hand, the sapphire is a chemically stable and relatively cheap substrate material for the growth of $\mathrm{ZnO} \mathrm{NW}$ arrays. However, two major issues need to be addressed for the $\mathrm{ZnO} \mathrm{NW}$ array on the sapphire substrate to be applied in optoelectronic devices: (1) since sapphire is an electrical insulator, the $\mathrm{ZnO} \mathrm{NWs}$ grown directly on the sapphire substrate are not electrically connected. Efficient electrical assembly of the fabricated $\mathrm{ZnO} \mathrm{NWs}$ is required. (2) The epitaxial mechanism of the high-quality $\mathrm{ZnO}$ on the sapphire substrate is not fully clarified due to the large in-plane lattice mismatches existing between the $\mathrm{ZnO}$ and the sapphire substrate ${ }^{4}$. The understanding of the epitaxial mechanism of the $\mathrm{ZnO}$ on the sapphire is preferred to achieve a reliable growth of the high-quality $\mathrm{ZnO}$ crystals.

In this work, a nanowire array on a film structure consisting of dense and vertically aligned $\mathrm{ZnO}$ NWs on a thick $\mathrm{ZnO}$ film was synthesized on an $a$-plane sapphire substrate through a one-step CVD process. This simple CVD process enables the growth of a dense $\mathrm{ZnO} \mathrm{NW}$ array on a thick $\mathrm{ZnO}$ film in the wurtzite-phase in a single-domain texture and highly oriented along the $\mathrm{ZnO} c$-axis direction over

\footnotetext{
4 The ideal $c$ surface of $\mathrm{ZnO}$ is six-fold symmetric while the ideal $a$ surface of sapphire is twofold symmetric, and thus they are essentially incompatible for epitaxial growth. On the lattice mismatch point of view, the $\mathrm{ZnO} a$ axis and the sapphire $c$ axis are related by a factor of four, giving a small lattice mismatch of about $0.076 \%$. However, the lattice mismatch on the other in-plane axis between $\mathrm{ZnO}[1 \overline{100}]$ and sapphire [1 100$]$ is large, reaching about $32 \%$. Thus, a residual stress may exist at the interface of the $\mathrm{ZnO} c$ surface and the sapphire $a$ surface, leading to the presence of misfit dislocations in the epi-grown $\mathrm{ZnO}$ film, which makes the epitaxial mechanism of high-quality $c$-oriented $\mathrm{ZnO}$ grown on the $a$-plane sapphire not fully understood.
}

a large area, as evidenced from the obtained transmission electron microscopy (TEM) images, selected area electron diffraction (SAED) patterns, x-ray diffraction (XRD) pole figures and XRD rocking curves. The epitaxial mechanism of this single-domain texture and $c$-oriented $\mathrm{ZnO} \mathrm{NW}$ array on a $\mathrm{ZnO}$ film structure is elucidated: a single crystalline $\mathrm{ZnAl}_{2} \mathrm{O}_{4}$ buffer layer was found to be formed between the epi-ZnO film and the $a$-plane sapphire substrate. The well-oriented single crystal $\mathrm{ZnAl}_{2} \mathrm{O}_{4}$ buffer layer (with aligned crystallographic orientations of $\mathrm{Al}_{2} \mathrm{O}_{3}$ [11 $\left.\left.\overline{2} 0\right]\left\|\mathrm{ZnAl}_{2} \mathrm{O}_{4}[0 \overline{2} 1]\right\| \mathrm{ZnO}[0001]\right)$ provides an improved in-plane symmetry and reduced lattice mismatches, and, therefore favors the epitaxial growth of a high-quality $\mathrm{ZnO} \mathrm{NWs}$ on a $\mathrm{ZnO}$ film structure. The understanding of the role of the $\mathrm{ZnAl}_{2} \mathrm{O}_{4}$ buffer layer in supporting the epitaxial growth of $\mathrm{ZnO}$ on the $a$-plane sapphire may have a profound impact on the growth techniques of single-domain and highly $c$-oriented $\mathrm{ZnO}$ films and nanostructures.

The optical and electronic properties of the fabricated $\mathrm{ZnO} \mathrm{NW}$ array on a $\mathrm{ZnO}$ film structure were further characterized by low-temperature photoluminescence (PL) spectroscopy and electrochemical impedance spectroscopy. Free exciton and bound exciton emissions along with phonon replica emissions were clearly observed in the low-temperature PL spectrum, indicating a good crystalline quality of the fabricated $\mathrm{ZnO} \mathrm{NW}$ array on a $\mathrm{ZnO}$ film structure. An n-type semiconductor behavior and the carrier density of $\sim 10^{17} \mathrm{~cm}^{-3}$ were obtained from the electrochemical impedance analysis. Finally, the fabricated $\mathrm{ZnO} \mathrm{NW}$ array on a $\mathrm{ZnO}$ film sample resulted in being an ideal template for a subsequent synthesis of a single crystal $\mathrm{ZnO}-\mathrm{ZnGa}_{2} \mathrm{O}_{4}$ core-shell NW array. The core-shell NW array on a film sample exhibited an enhanced anticorrosive and photo-oxidative performance when used as a photoanode in a photoelectrochemical water splitting cell. The one-step CVD technique is suitable for the large-scale fabrication of a single-domain and highly $c$-oriented $\mathrm{ZnO} \mathrm{NW}$ array on a $\mathrm{ZnO}$ film structure and will contribute to the development of $\mathrm{ZnO}$-based high-performance electronic, optical and optoelectronic devices.

\section{Experimental details}

The $\mathrm{ZnO}$ nanostructure of a dense $\mathrm{ZnO}$ NW array on a thick $\mathrm{ZnO}$ film was fabricated on $a$-plane sapphire substrates over a large area using a simple CVD process. An Au-coated $a$-plane sapphire was used as substrate and placed at the center of the CVD furnace tube. $\mathrm{ZnO}$ powder and graphite powder ( $0.2 \mathrm{~g}$ in weight) with a $2: 1$ weight ratio were loaded into an alumina boat as precursors and placed at a position $1 \mathrm{~cm}$ upstream from the substrate in the furnace tube. The furnace operated at $1000^{\circ} \mathrm{C}$ for $30 \mathrm{~min}$ under argon and oxygen atmosphere (5:1 in volume ratio) flowing through at a working pressure of $50 \mathrm{mbar}$. Then the furnace cooled down to room temperature naturally. A dense $\mathrm{ZnO} \mathrm{NW}$ array on a thick $\mathrm{ZnO}$ film structure was fabricated on the Au-coated $a$-plane sapphire substrate. 

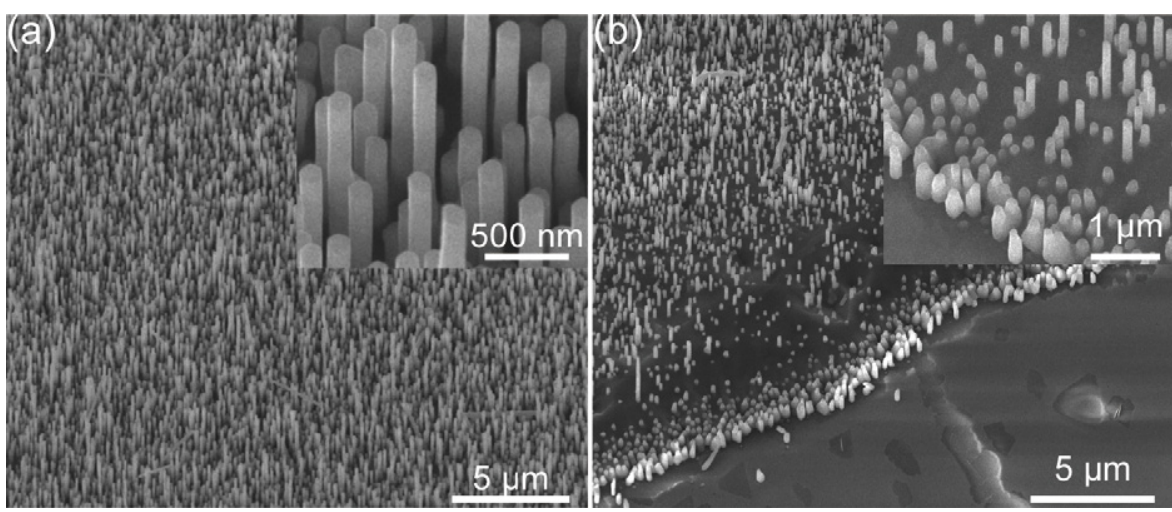

Figure 1. The morphology of the fabricated $\mathrm{ZnO}$ nanowire array on a $\mathrm{ZnO}$ film. (a) Tilted-angle SEM image of large-area dense and vertically aligned $\mathrm{ZnO}$ nanowires on the Au-coated $a$-plane sapphire substrate. Inset in (a) is a close-up of the vertically aligned $\mathrm{ZnO}$ nanowires. (b) Tilted-angle SEM image showing the structure of $\mathrm{ZnO}$ nanowires standing on the $\mathrm{ZnO}$ film at the edge area of the Au-coated $a$-plane sapphire surface. Inset in (b) is a close-up of the $\mathrm{ZnO}$ nanowire on a $\mathrm{ZnO}$ film structure at the Au-coated edge area.

Scanning electron microscopy (SEM) images of the fabricated $\mathrm{ZnO} \mathrm{NW}$ array on a thick $\mathrm{ZnO}$ film sample were registered with a Hitachi S3000N microscope. Standard $\theta / 2 \theta$ scans, the $\mathrm{x}$-ray diffraction rocking curves and $\mathrm{x}$-ray pole figures measurements of the fabricated $\mathrm{ZnO}$ structures were made using the Smart lab XRD of Rigaku, Japan. TEM and STEM observations were carried out with JEOL JEM-2100F and ARM-200F microscopes and a JED-2300T EDS system. HAADF STEM images were acquired with the probe forming an aperture semiangle of $\sim 22 \mathrm{mrad}$ and an inner detection angle greater than 70 mrad.

The electrochemical impedance measurements of the synthesized $\mathrm{ZnO} \mathrm{NW}$ array on a $\mathrm{ZnO}$ film sample were performed with a Princeton Applied Research VersaSTAT 4 potentiostat. An electrolyte solution of $0.5 \mathrm{M} \mathrm{NaClO}_{4}$ was prepared and buffered to a $\mathrm{pH}$ of 7.0 with the phosphate buffer solution. $\mathrm{An} \mathrm{Ag} / \mathrm{AgCl}$ electrode in the saturated $\mathrm{KCl}$ solution was used as a reference electrode, a $\mathrm{Pt}$ wire was used as a counter electrode and the $\mathrm{ZnO} \mathrm{NW}$ array on a $\mathrm{ZnO}$ film sample was used as a working electrode. Before the electrochemical impedance measurement, $\mathrm{N}_{2}$ gas was bubbled for $10 \mathrm{~min}$ to get rid of the $\mathrm{O}_{2}$ in the electrolyte. The cyclic voltammetry and the Nyquist plots were at first registered in the dark in order to determine the appropriate range for the applied bias and the frequency applied to the electrode of the ZnO NW sample. Then, the Mott-Schottky analysis was performed in the dark with a bias varying from -0.4 to $0.4 \mathrm{~V}$ (versus the reference electrode) and at a frequency of $1 \mathrm{kHz}$.

\section{Results and discussions}

\subsection{Morphology and texture quality of the $\mathrm{ZnO}$ nanowire array on a $\mathrm{ZnO}$ film}

The morphology and structure of the synthesized $\mathrm{ZnO} \mathrm{NW}$ array on a $\mathrm{ZnO}$ film are presented in the tilted-angle scanning electron microscopy (SEM) images. Figure 1(a) shows a highly uniform and densely assembled $\mathrm{ZnO} \mathrm{NW}$ array grown over a large area on an Au-coated $a$-plane sapphire substrate.
The inset in figure 1(a) is an enlarged SEM image of the vertically aligned $\mathrm{ZnO}$ NWs. The vertical alignment and smooth sidewalls of the $\mathrm{ZnO}$ NWs can be clearly observed. The average diameter of the $\mathrm{ZnO} \mathrm{NW}$ is about $100 \mathrm{~nm}$ and the average length is about $1 \mu \mathrm{m}$. Figure $1(\mathrm{~b})$ shows the morphology of the $\mathrm{ZnO} \mathrm{NW}$ array on a $\mathrm{ZnO}$ film sample near the Au-coated edge area of the $a$-plane sapphire surface. The density of the NWs is low near the edge area so the underlying $\mathrm{ZnO}$ film can be noticed. Thus we can infer that the roots of the NWs are connected to the $\mathrm{ZnO}$ film underneath. Energy dispersive $\mathrm{x}$-ray analyses (EDX) performed on the underlying $\mathrm{ZnO}$ film and on the NW array gave similar results: a $\mathrm{Zn} / \mathrm{O}$ atomic ratio of about $1 / 1$ was obtained, indicating that the NWs and the underlying film are made of $\mathrm{ZnO}$.

Scanning transmission electron microscopy (STEM) was used to analyze the structure and the composition of the $\mathrm{ZnO} \mathrm{NW}$ array on a $\mathrm{ZnO}$ film sample. Figure 2(a) shows a cross-sectional high-angle annular dark-field (HAADF) STEM image of the NW array on a $\mathrm{ZnO}$ film structure. The average length of the NWs is about $1 \mu \mathrm{m}$ and the thickness of the film is about $2.5 \mu \mathrm{m}$. Energy dispersive spectroscopy (EDS) analyses were performed in STEM to examine the spatial distribution of the various chemical elements in the studied sample. The STEM-EDS mapping images presented in figure 2(b) show that the signal from the $\mathrm{Al}$ is confined in the substrate region and the signal from the $\mathrm{Zn}$ is confined in the nanowires and film region above the substrate, confirming that the structure of a $\mathrm{ZnO} \mathrm{NW}$ array on a $\mathrm{ZnO}$ film is synthesized on the $a$-plane sapphire substrate. A magnified bright-field STEM image and STEM-EDS mapping images on the top region of the sample (see figure 2(c)) reconfirm the formation of vertically aligned $\mathrm{ZnO} \mathrm{NWs}$ on the $\mathrm{ZnO}$ film.

Electron diffraction (ED) analyses in transmission electron microscopy (TEM) were further performed to characterize the crystalline quality and the crystallographic orientation of the $\mathrm{ZnO} \mathrm{NW}$ array on a $\mathrm{ZnO}$ film sample. The ED pattern taken in the $\mathrm{ZnO}$ film region along the [1100] zone axis (see figure 2(d)) shows that the $\mathrm{ZnO}$ thick film is single crystalline and well aligned along the [0001] direction of the wurtzite-phase. This feature can also be observed from the ED 
(c)
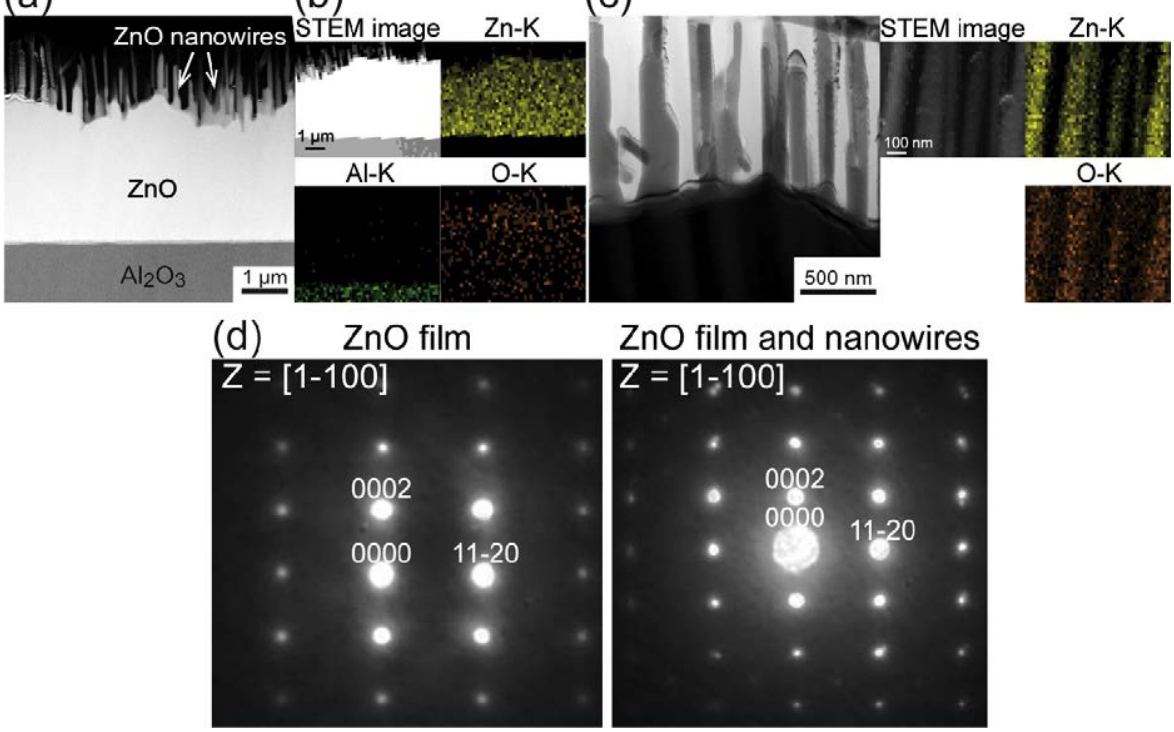

$\mathrm{ZnO}$ film and nanowires

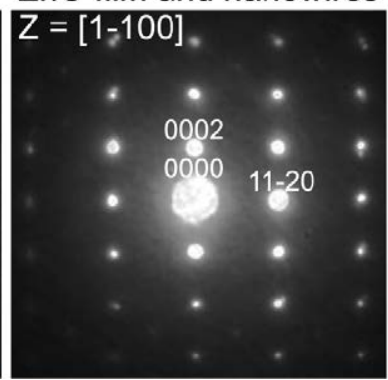

Figure 2. (a) HAADF STEM image of a cross-sectional view of the $\mathrm{ZnO}$ nanowire array on a $\mathrm{ZnO}$ film structure. (b) HAADF STEM image and EDS mapping images of the $\mathrm{ZnO}$ nanowire array on a $\mathrm{ZnO}$ film structure on the $a$-plane sapphire substrate. (c) Magnified bright-field STEM image of the ZnO nanowire array region (left); HAADF STEM image and Zn, O EDS mapping images of the ZnO nanowire array region (right). (d) Electron diffraction patterns taken in the region of the $\mathrm{ZnO}$ film (left) and in the region containing the $\mathrm{ZnO}$ film with $\mathrm{ZnO}$ nanowires (right).

pattern taken in the region containing the $\mathrm{ZnO}$ film and $\mathrm{ZnO}$ NWs (see figure 2(d)).

Since the ED technique is a local area analysis and provides information of the examined crystals within a small region defined by the TEM observation aperture (usually in the nanometer/micrometer range), it may happen that it does not represent the microstructure of the sample on a large scale. Thus a standard $\mathrm{X}$-ray diffraction (XRD) $\theta / 2 \theta$ measurement was performed to examine the crystal structure and crystal plane orientations on a millimeter range. The XRD result presented in figure 3(a) shows two dominant diffraction peaks indexed as the wurtzite $\mathrm{ZnO}(0002)$ and (0004) planes. This result is evidence for the good crystallinity of the $\mathrm{ZnO}$ film and $\mathrm{ZnO} \mathrm{NWs}$ and for their preferential growth orientation along the $\mathrm{ZnO}[0001]$ direction. The XRD $\theta$ rocking curve measurement of the $\mathrm{ZnO}(0002)$ peak is reported in figure $3(\mathrm{~b})$. A small value of the full width at half maximum of $0.097^{\circ}$ is obtained, indicating a perfect alignment of the $\mathrm{ZnO}$ crystalline domains in the [0001] direction.

We have demonstrated that the $\mathrm{ZnO} \mathrm{NW}$ array on a $\mathrm{ZnO}$ film sample is of a single crystalline quality and well aligned along the $\mathrm{ZnO}[0001]$ crystallographic axis. However, a rotational degree of freedom around the $\mathrm{ZnO}[0001]$ axis has to be characterized [17]. Indeed, the texture of the sample could be either a unique crystal domain or a fiber domain. In the fiber domain-type structure, the orientations of the crystallographic axes normal to [0001] axis are not fixed and result in a fiber-like arrangement. The texture quality of the material is an important characteristic, determining its optical, optoelectronic, and mechanical properties [10, 17] (see footnote 4). To clarify the texture type of the fabricated NWs on a $\mathrm{ZnO}$ film sample, $\mathrm{x}$-ray pole figure analysis was carried out. The $\mathrm{x}$-ray pole figure measurement shown in figure 3(c) was performed on the $\mathrm{ZnO} \mathrm{NW}$ array on a $\mathrm{ZnO}$ film sample with the $\mathrm{ZnO}(10 \overline{1} 2)$ reflection. The scheme of the stereographic projection of the X-ray pole figure measurements is shown in figure 3(c). An alternative way to demonstrate the resulting pole figure is the azimuthal $\beta$ scan curve covering the radial angle $\alpha$ from $0^{\circ}$ to $90^{\circ}$ presented in figure 3(c). A strong reflection intensity in the pole figure measurement is observed when the Euler angles $(\theta, \alpha, \beta)$ of a sample placed in the goniometer satisfy the diffraction condition of its crystal structure [17]. A six-fold rotational symmetry of the $(10 \overline{1} 2)$ reflections can clearly be seen in the resulting pole figure, implying that no (or very few) in-plane rotational domains or twins were present in the fabricated $\mathrm{NW}$ array on a $\mathrm{ZnO}$ film sample. Direct evidence for the single crystal domain of the $\mathrm{ZnO} \mathrm{NW}$ array on a $\mathrm{ZnO}$ film sample is provided by the SEM top-view image shown in figure $3(\mathrm{~d})$. It can be clearly noticed that the tips of the $\mathrm{ZnO}$ NWs form hexagons whose sides are perfectly aligned. Such an alignment of the hexagons can only be obtained if all ZnO NWs are homoepitaxially grown on a single crystalline domain $\mathrm{ZnO}$ film. It can also be noted that the hexagonal alignment is obtained over a large area. Thus, our synthesized sample of the $\mathrm{ZnO} \mathrm{NW}$ array on a $\mathrm{ZnO}$ film is composed of a unique $\mathrm{ZnO}$ crystal domain, described by the texture orientation of $\{(0001)[10 \overline{10}]\}$, as depicted in figure 3(d).

\subsection{Epitaxial growth mechanism of the $\mathrm{ZnO}$ nanowire array on a $\mathrm{ZnO}$ film}

The heteroepitaxy of the $\mathrm{ZnO}$ film on the $a$-plane sapphire substrate is investigated in order to understand the growth mechanism of the $\mathrm{ZnO}$ epi-layer and to achieve a better control of the fabrication of high-quality and efficient $\mathrm{ZnO}$ 
(a)
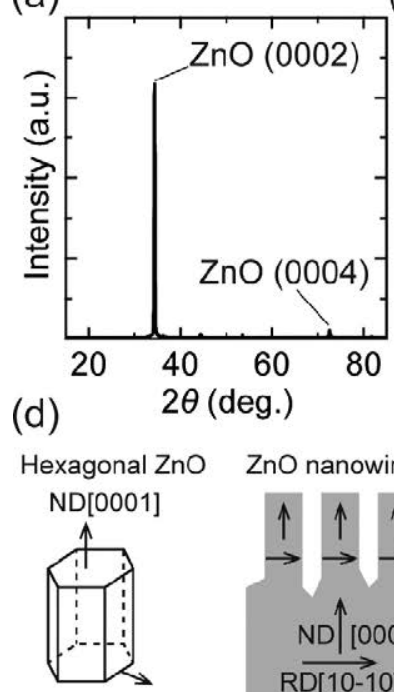

$\mathrm{RD}[10-10]$ (b)

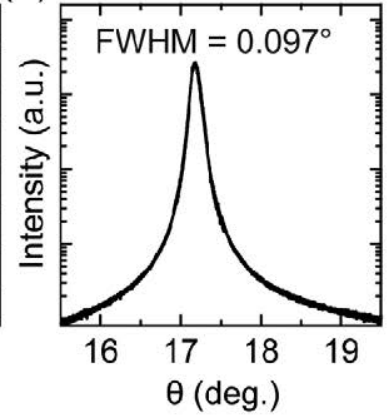

(c)

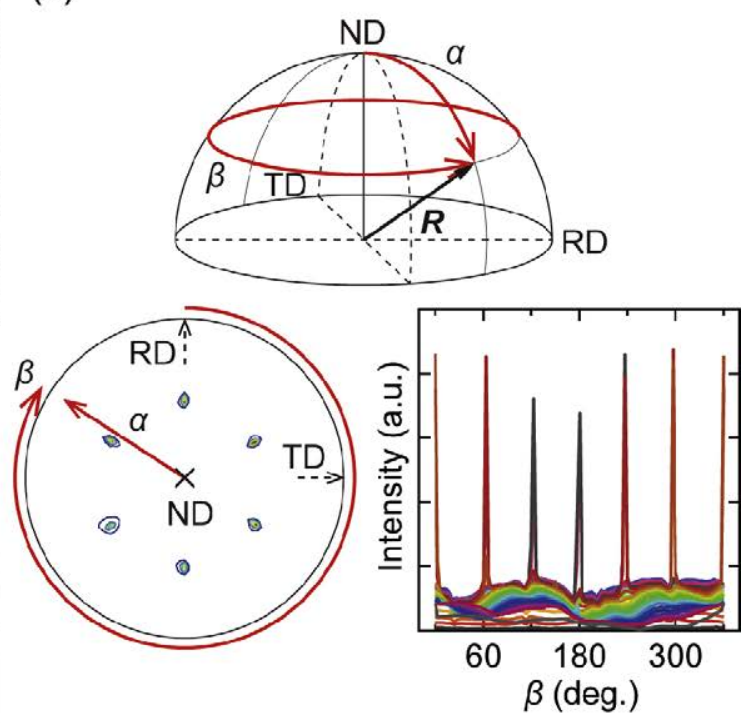

Figure 3. XRD analyses of the $\mathrm{ZnO}$ nanowire array on a $\mathrm{ZnO}$ film sample. (a) Standard $\mathrm{XRD} \theta / 2 \theta$ measurement. (b) Rocking curve (intensity versus $\theta$ ) around the $\mathrm{ZnO}(0002)$ diffraction peak. (c) Schematic drawing of the $\mathrm{x}$-ray pole figure measurement (left); stereographic projections of the $\mathrm{x}$-ray pole figure results for the $\mathrm{ZnO}(10 \overline{1} 2)$ reflection (middle), color areas indicate higher diffraction intensity; azimuthal $\beta$ curves of the $\mathrm{x}$-ray pole figure result (right). ND: north-pole direction or normal direction; RD: rotational direction; TD: transverse direction. (d) Schematic of the $\mathrm{ZnO}$ crystal (left) and the $\mathrm{ZnO}$ nanowire array on a $\mathrm{ZnO}$ film having a unique crystal domain orientation (middle); top-view SEM image of the ZnO nanowires; a perfect alignment of the nanowires' hexagonal tips can be clearly seen (right).

nanostructures. As the problems of the unmatched geometry and a large one-axis lattice mismatch exist between the $\mathrm{ZnO} c$-plane and the sapphire $a$-plane (see footnote 4 ), the epitaxial growth of a high-quality $\mathrm{ZnO}$ films or NWs on the sapphire substrates is not fully clarified. Therefore, the investigation of the interface between the $\mathrm{ZnO}$ epi-layer and the $a$-plane sapphire substrate is of great importance in order to understand the heteroepitaxial growth of a high-quality $\mathrm{ZnO}$ films or NWs on the $a$-plane sapphire.

In the HAADF STEM image of figure 2(a), an intermediate layer showing a different brightness contrast can be noticed between the $\mathrm{ZnO}$ film and the $a$-plane sapphire substrate. Note that in HAADF STEM imaging, the brightness in the image depends on the atomic number $Z$ of the elements making up the sample [18]. A magnified HAADF STEM observation was further performed at the $\mathrm{ZnO}$ and the $a$-plane sapphire interface region, as shown in figure 4(a). The brightness of the intermediate layer is found to be in between that of the $\mathrm{ZnO}$ film and the sapphire, revealing that the $0.08 \mu \mathrm{m}$-thick intermediate layer is likely a diffusion layer having a different chemical composition. The Al, Zn, $\mathrm{O}$ K-shell EDS mapping images presented in figure 4(b) confirm that the intermediate layer contains $\mathrm{Zn}, \mathrm{Al}$, and $\mathrm{O}$ elements. Further, the ED pattern of the interface region shown in figure 4(d) taken along the zone axis of [112] gives two sets of single crystalline electron diffraction dots, identified as the spinel $\mathrm{ZnAl}_{2} \mathrm{O}_{4}$ and the sapphire. Hence, the intermediate layer is composed of a single crystalline spinel $\mathrm{ZnAl}_{2} \mathrm{O}_{4}$ with a $[0 \overline{2} 1]$ crystallographic orientation, parallel to the [0001] direction of the $\mathrm{ZnO}$ film and parallel to the [11 20$]$ (see footnote 4) direction of the $a$-plane sapphire substrate, as illustrated in figure $4(\mathrm{c})$. Note that the $(0 \overline{2} 1)$ plane of the spinel $\mathrm{ZnAl}_{2} \mathrm{O}_{4}$ has a trigonal crystalline symmetry that matches the hexagonal geometry of the wurtzite-phase $\mathrm{ZnO}$ $c$-plane and exhibits small lattice mismatches of $1.5 \%$ and $0.47 \%$ with the two crystallographic axes of the $\mathrm{ZnO} c$-plane. In section 3.3, we discuss the possible growth mechanism of the high-quality and [0001] oriented $\mathrm{ZnO}$ film on the $a$-plane sapphire. An uniaxial heteroepitaxy first occurred to form a single orientational $\{(0001)[10 \overline{10}]\} \mathrm{ZnO}$ domain, due to the perfectly matched lattice constants between the $\mathrm{ZnO} a$-axis and the sapphire substrate $c$-axis. The anisotropic nature of the crystal lattice in the $a$-plane of the sapphire helps to reduce the formation of the rotational domains within $\mathrm{ZnO}(0001)$ plane in the beginning of the growth process, as has been reported in the literature for the selected growth routes [10]. However, the large lattice mismatch induced in-plane stress in the axial direction between the $\mathrm{ZnO}[1 \overline{100}]$ and the sapphire [1 $1 \overline{100}$ ] becomes difficult to balance during the growth process. During our CVD growth of the $\mathrm{ZnO}$ NWs on a $\mathrm{ZnO}$ film sample, diffusion of $\mathrm{Zn}$ atoms into the sapphire substrate occurs, resulting in a formation of a single crystalline and $[0 \overline{2} 1]$ oriented $\mathrm{ZnAl}_{2} \mathrm{O}_{4}$ intermediate layer. Thus, an optimized geometry of $\mathrm{ZnO}(0001)\left\|\mathrm{ZnAl}_{2} \mathrm{O}_{4}(0 \overline{2} 1)\right\|$ $\mathrm{Al}_{2} \mathrm{O}_{3}(1120)$ with an improved lattice matching, helps to reduce the residual stress at the $\mathrm{ZnO}$-sapphire interface and supports the growth of a high-quality and [0001] oriented $\mathrm{ZnO}$ film. The formation of the $\mathrm{ZnAl}_{2} \mathrm{O}_{4}$ buffer layer is made possible by a diffusion reaction which requires a temperature of about $600^{\circ} \mathrm{C}$ [19-21], a condition readily fulfilled under the growth conditions we apply (the temperature of the furnace is of about $1000^{\circ} \mathrm{C}$ ).

Note that the $\mathrm{ZnO} \mathrm{NW}$ array on a $\mathrm{ZnO}$ film structure is only grown on the Au-coated surface area of the $a$-plane 


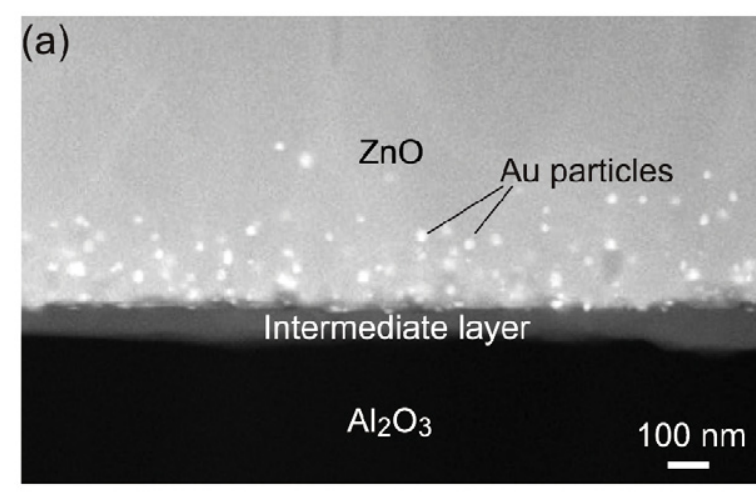

(c)

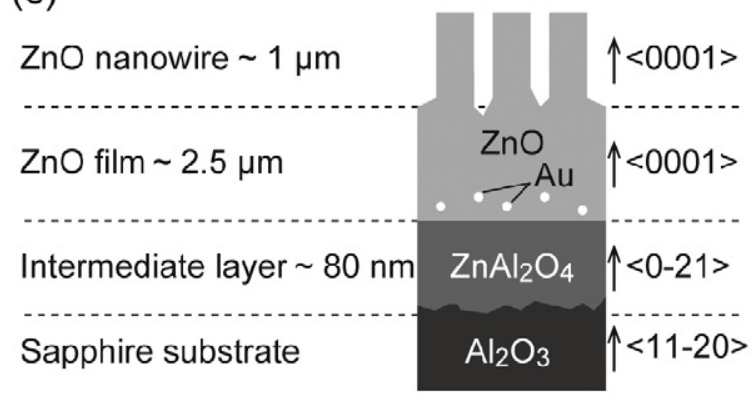

(b)

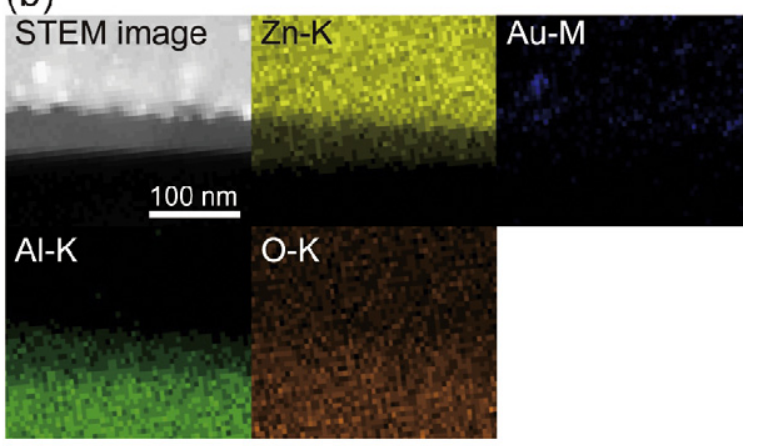

(d)

$\mathrm{ZnAl}_{2} \mathrm{O}_{4}$ intermediate layer region

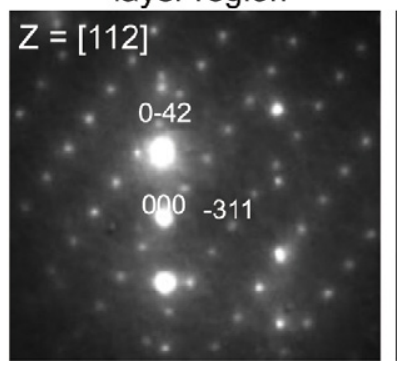

Sapphire substrate

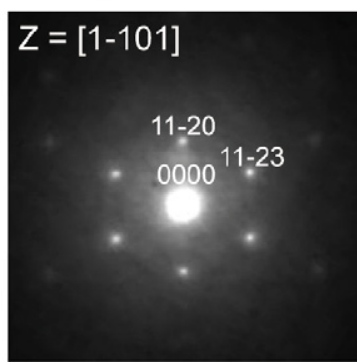

Figure 4. (a) HAADF STEM image of a magnified view at the interface of the $\mathrm{ZnO}$ film and the $a$-plane sapphire substrate. (b) HAADF STEM image and EDS mapping images taken in the interface region of the ZnO film and the sapphire substrate. (c) Schematic illustration of the $\mathrm{ZnO}$ nanowire array on a $\mathrm{ZnO}$ film structure showing aligned crystallographic orientations within each layer. (d) Electron diffraction patterns taken in the interface region of the $\mathrm{ZnO}$ film and the $a$-plane sapphire substrate and taken in the region of the $a$-plane sapphire substrate.

sapphire substrate, which indicates that the nucleation of $\mathrm{ZnO}$ on the surface of $a$-plane sapphire is difficult without the assistance of $\mathrm{Au}$ under our growth conditions. As reported [3-6], Au is an efficient catalyst which captures and localizes $\mathrm{Zn}$ and $\mathrm{O}$ vapor atoms, and thus contributes to the formation of $\mathrm{ZnO}$ nuclei in a conventional vapor-liquid-solid (VLS) process. This is due to a strong binding affinity between the $\mathrm{Au}$ atoms and $\mathrm{ZnO}$ [22]. Therefore, a schematic of the proposed growth process of our $\mathrm{ZnO}$ nanowire array on a film structure is shown in figure 5. In figure 5(a), the $a$-plane sapphire substrate was first deposited with Au particles by a quick coater. The sizes of the Au particles deposited on the $a$-plane sapphire substrate was measured using a dynamic force microscope (DFM) of SII (SPI 3800N/SPA-400). The estimated diameters of the as-deposited Au particles was about $10 \mathrm{~nm}$ (see the DFM image in figure 5(e)). With the increase of temperature in the CVD growth process, the Au particles on the $a$-plane sapphire substrate tended to migrate together to form large particles, as shown in figure 5(b). To prove the formation of large Au particles at elevated temperatures, an annealing experiment was performed. An $a$-plane sapphire deposited with the same amount of Au was annealed at $1000^{\circ} \mathrm{C}$ for $15 \mathrm{~s}$ with Ar gas flowed through and without $\mathrm{Zn}$ and $\mathrm{O}$ sources at a pressure of 50 mbar. After the annealing process, the Au-deposited $a$-plane sapphire substrate cooled down to room temperature naturally. The SEM image of the annealed sample is shown in figure 5(e). Large Au particles were formed after the annealing process with estimated diameters of about $75 \mathrm{~nm}$ and $25 \mathrm{~nm}$. In our $\mathrm{ZnO}$ growth process with the $\mathrm{Zn}$ and $\mathrm{O}$ sources, the enlarged Au particles captured the $\mathrm{Zn}$ and $\mathrm{O}$ vapor to form $\mathrm{Au}-\mathrm{Zn}$ alloy particles at elevated temperatures and serves as a catalyst to facilitate the $\mathrm{ZnO}$ nucleation as shown in figure 5(c). Due to the presence of a sufficient supply of Zn and $\mathrm{O}$ vapor sources, a fast growth of the $\mathrm{ZnO}$ nanowires and the $\mathrm{ZnO}$ film occurred. It is suggested that the large $\mathrm{Au}-\mathrm{Zn}$ alloy particles could meet the minimum diameter requirement for the growth of nanowire in a VLS growth process [23], and supported the growth of the $\mathrm{ZnO}$ nanowires. During the growth of the $\mathrm{ZnO}$ nanowires, Au may migrate away from its original location because Au has a high diffusion rate at high growth temperature [4, 24, 25]. In our process, it is very probable that $\mathrm{Au}$ atoms diffused from the Au-rich area to the area with low $\mathrm{Au}$ density on the surface of the $\mathrm{ZnO}$ nanowires and $\mathrm{ZnO}$ film. In consequence, the $\mathrm{ZnO}$ nanowires stopped the growth without the sufficient support of Au catalysts, and, therefore a Au-catalyzed VLS growth process explained the growth of the $\mathrm{ZnO}$ nanowires. In addition, a large amount of Au particles with smaller diameters were also formed on the surface of the $a$-plane sapphire substrate at the initial stage of the high-temperature CVD process. The smaller Au particles captured the $\mathrm{Zn}$ and $\mathrm{O}$ sources to form $\mathrm{ZnO}$ nuclei and supported the growth of the $\mathrm{ZnO}$ film. This hypothesis is confirmed with the STEM and EDS analyses of the fabricated $\mathrm{ZnO}$ sample. Many Au particles with diameters of about $10-30 \mathrm{~nm}$ were buried at the very bottom of the $\mathrm{ZnO}$ film, 
(a)

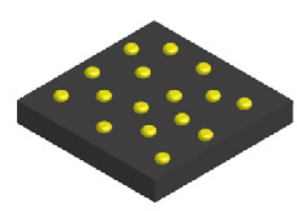

(b)

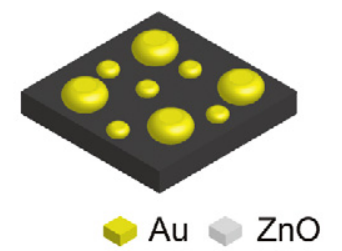

(c)

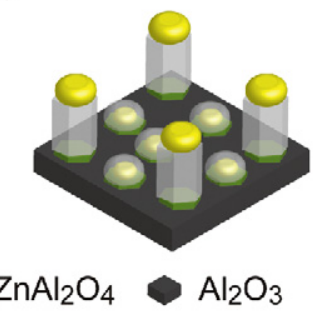

(d)

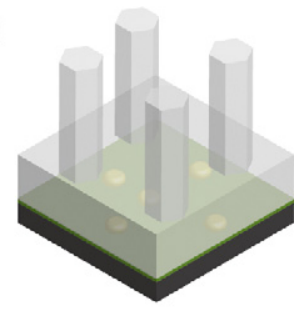

(e) DFM image (left) and SEM image of the Au-coated a-plane sapphire

before annealing

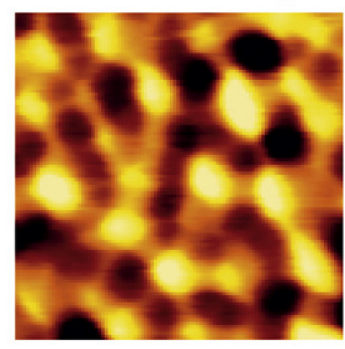

$10 \mathrm{~nm}$ after annealing

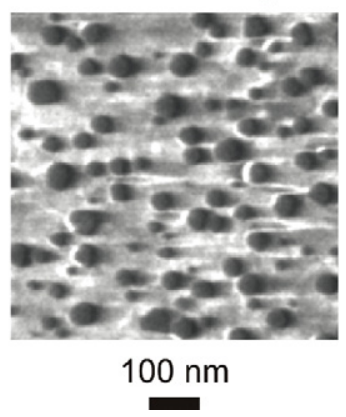

Figure 5. (a)-(d) Schematic drawing of the CVD growth process of the $\mathrm{ZnO}$ nanowire array on a $\mathrm{ZnO}$ film structure on the $a$-plane sapphire substrate. (a) Au particles was deposited on the $a$-plane sapphire substrate. (b) Large Au particles were formed at elevated temperatures during the CVD process. (c) Au particles captured $\mathrm{Zn}$ and $\mathrm{O}$ vapor atoms to form the $\mathrm{ZnO}$ nuclei. The large droplets supported the growth of the $\mathrm{ZnO}$ nanowires and the small droplets supported the growth of the $\mathrm{ZnO}$ film. The $\mathrm{ZnAl}_{2} \mathrm{O}_{4}$ buffer layer was also formed at the interfaces between the $\mathrm{ZnO}$ and the sapphire substrate. (d) The $\mathrm{ZnO}$ nanowire array on a $\mathrm{ZnO}$ film structure was formed after the CVD growth. (e) DFM and SEM images of the Au particles on the $a$-plane sapphire substrate before and after an annealing process. The annealing process of the Au-coated $a$-plane sapphire substrate was operated at $1000^{\circ} \mathrm{C}$ for $15 \mathrm{~s}$ with only argon gas flowed through at a pressure of $50 \mathrm{mbar}$.

as shown in the HAADF STEM image of figure 4(a). Au nanoparticles are detected as strong brightness contrast in figure 4(a) due to the large difference in $Z$ between $\mathrm{Au}$ and other elements [18]. The presence of Au nanoparticles in the vicinity of the interface on the $\mathrm{ZnO}$ film was further confirmed by $\mathrm{Au} \mathrm{M}$-shell EDS analysis in figure 4(b). After the CVD growth process, the $\mathrm{ZnO}$ nanowire array on a film structure was formed on the $a$-plane sapphire substrate, as shown in figure $5(d)$.

The low-temperature PL spectrum excited with a $\mathrm{He}-\mathrm{Cd}$ UV laser operating at $325 \mathrm{~nm}$ was measured at $13 \mathrm{~K}$ and permitted to characterize the optical quality of the studied structures. The PL spectrum presented in a semi-log scale in figure 6 shows one dominant peak emission in the 3.35-3.36 eV region. This sharp and intense peak is attributed to the neutral-donor-bound exciton line $\left(\mathrm{D}^{0} \mathrm{X}\right)$ of $\mathrm{ZnO}$. On the high-energy side of this peak, the $\mathrm{ZnO} A$-free exciton at $\mathrm{FX}_{A}^{n=1}=3.37 \mathrm{eV}$ can be observed. The first excited state emission of the $A$-free exciton at $\mathrm{FX}_{A}^{n=2}=3.42 \mathrm{eV}$ can be observed unambiguously. The observation of the $A$-free exciton and its first excited state emission is clear evidence that the $\mathrm{ZnO} \mathrm{NW}$ array on a $\mathrm{ZnO}$ film sample is of a high crystalline quality and essentially free of strain. Furthermore, fine structures related to longitudinal optical (LO) phonon replica lines of the dominant neutral-donor-bound exciton $\mathrm{D}^{0} \mathrm{X}$ emission and the $A$-free exciton of $\mathrm{FX}_{A}^{n=1}$ are clearly seen in the spectrum presented in figure 6 . The first-order (1LO), second-order (2LO), and third-order (3LO) replicas of the $\mathrm{D}^{0} \mathrm{X}$ emission and the first-order (1LO) and second-order (2LO) replicas of the $A$-free exciton of $\mathrm{FX}_{A}^{n=1}$ are shifted by $\sim 70 \mathrm{meV}$ towards lower energies from the main $\mathrm{D}^{0} \mathrm{X}$ and $\mathrm{FX}_{A}^{n=1}$ emission peaks. The two-electron satellite transition of $\left(\mathrm{D}^{0} \mathrm{X}\right)_{2 \mathrm{e}}$, characteristic of the neutral-donor-bound exciton transition is identified at $\sim 30 \mathrm{meV}$ below the energy of the $\mathrm{D}^{0} \mathrm{X}$ emission peak. All the positions of the emission peaks are in good agreement with literature reports for high-quality $\mathrm{ZnO}$ films and/or 1D nanostructures [4, 26, 27]. The PL properties indicate that high-quality $\mathrm{ZnO} \mathrm{NWs}$ on a $\mathrm{ZnO}$ film were synthesized thanks to the formation of a single crystal $\mathrm{ZnAl}_{2} \mathrm{O}_{4}$ buffer layer between the sapphire substrate and the $\mathrm{ZnO}$ film, which helps to diminish the strain present in the $\mathrm{ZnO}$ layer due to the lattice constant mismatch between $\mathrm{ZnO}$ and the $a$-plane sapphire.

Electrochemical impedance spectroscopy was used to investigate the charge carrier density in the $\mathrm{ZnO} \mathrm{NW}$ array on a $\mathrm{ZnO}$ film sample. Typically, carrier density values ranging from $10^{16}$ to $10^{18} \mathrm{~cm}^{-3}$ are reported in the literature for the undoped $\mathrm{ZnO}$ films or nanostructures fabricated by the gas-phase deposition methods [28, 29]. For the aqueous chemical deposition methods, an increased carrier density of $10^{19}-10^{20} \mathrm{~cm}^{-3}$ is usually observed, likely because of the unintentional doping of the $\mathrm{ZnO}$ NWs with hydrogen. This has been evidenced by the effect of the post-annealing treatment of the samples, in which the carrier density reduction to the range of $10^{16}-10^{18} \mathrm{~cm}^{-3}$ was 


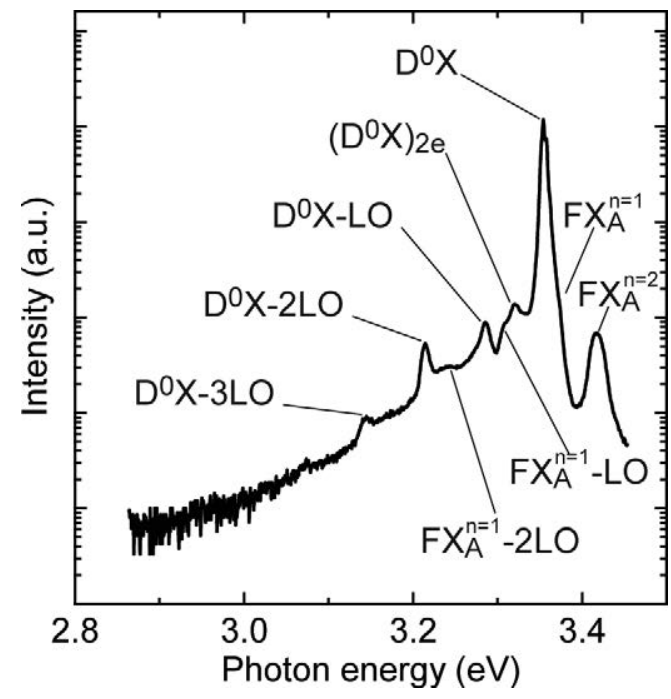

Figure 6. Low-temperature PL spectra performed at $13 \mathrm{~K}$ of the $\mathrm{ZnO}$ nanowire array on a $\mathrm{ZnO}$ film sample plotted in a semi-logarithmic scale.

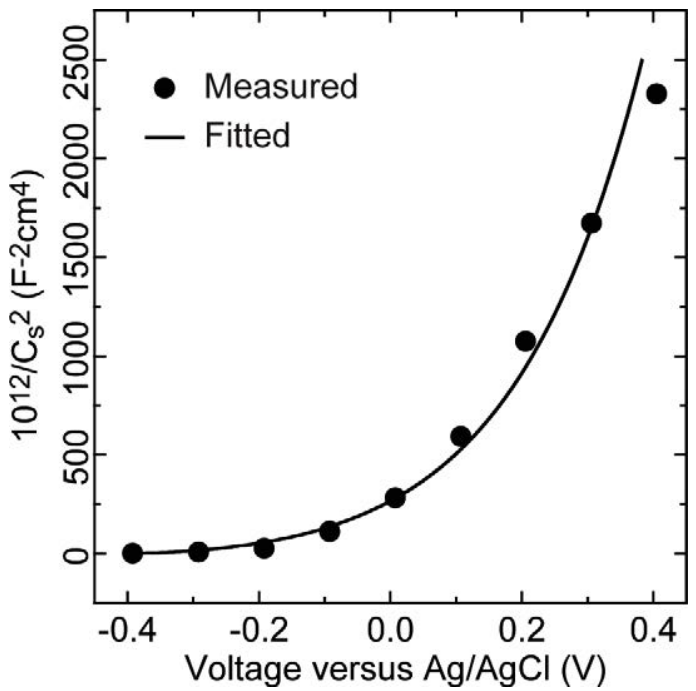

Figure 7. The Mott-Schottky plot of the $\mathrm{ZnO}$ nanowire on a $\mathrm{ZnO}$ film sample taken at a frequency of $1 \mathrm{kHz}$ under dark conditions for a bias voltage ranging from -0.4 to $0.4 \mathrm{~V}$ (versus $\mathrm{Ag} / \mathrm{AgCl}$ electrode)

observed due to the exodiffusion of the hydrogen during the post-annealing [30]. In our study, a calculated carrier density of about $10^{17} \mathrm{~cm}^{-3}$ with a n-type conductivity is obtained for the $\mathrm{ZnO} \mathrm{NW}$ array on a $\mathrm{ZnO}$ film sample via fitting of the Mott-Schottky data using the Mora-Sero model [30], as shown in figure 7.

\subsection{Photoelectrochemical applications of the $\mathrm{ZnO}$ nanowire array on a $\mathrm{ZnO}$ film}

To sum up, we demonstrate a cost-effective method for the fabrication of a dense $\mathrm{ZnO} \mathrm{NW}$ array on a $\mathrm{ZnO}$ film structure having a single crystal domain quality on the $a$-plane sapphire substrate. This technique can be applied to large-scale fabrication of high-quality $\mathrm{ZnO} \mathrm{NW}$ arrays and may substantially reduce the cost and make easier the integration of $\mathrm{ZnO} \mathrm{NW}$ arrays in electronic, optoelectronic, electrochemical and electromechanical applications. In addition, the $\mathrm{ZnO} \mathrm{NW}$ array on a $\mathrm{ZnO}$ film structure is an ideal host to realize a broad range of functional materials by post-process doping or hetero-growth in the radial direction of the NWs, forming a $\mathrm{ZnO}$-based core-shell nanowire array on a film structure. The usefulness of this nanowire array on a film structure is dictated by the realization of an efficient electrical charge collection because the nanowire and the film are made of same material with high crystal quality.

In the following, we used the $\mathrm{ZnO} \mathrm{NW}$ array on a $\mathrm{ZnO}$ film sample as a template for the fabrication of a dense $\mathrm{ZnO}-\mathrm{ZnGa}_{2} \mathrm{O}_{4}$ core-shell $\mathrm{NW}$ array on a $\mathrm{ZnO}$ film in the second CVD growth process. The synthesized $\mathrm{ZnO}-\mathrm{ZnGa}_{2} \mathrm{O}_{4}$ core-shell NW array on a $\mathrm{ZnO}$ film sample permits an easy charge carrier collection through its thick bottom conductive film and has a stable and efficient photo-oxidation ability when used as a photoanode in a photoelectrochemical (PEC) water splitting cell [6]. The $\mathrm{ZnO}-\mathrm{ZnGa}_{2} \mathrm{O}_{4}$ core-shell NWs are perfectly arranged, tightly packed and bonded altogether at their bases through the same material over large areas, as shown in the SEM image presented in figure 8(a). The $\mathrm{ZnO}-\mathrm{ZnGa}_{2} \mathrm{O}_{4}$ core-shell NWs are single crystalline, with their crystallographic orientations aligned along $\mathrm{ZnGa}_{2} \mathrm{O}_{4}$ [111] \| $\mathrm{ZnO}[0002]$ [31].

The PEC performance of the $\mathrm{ZnO}-\mathrm{ZnGa}_{2} \mathrm{O}_{4}$ core-shell nanostructured photoanode was investigated in a nonsacrificial agent of a $0.1 \mathrm{M} \mathrm{Na}_{2} \mathrm{SO}_{4}$ electrolyte solution at $\mathrm{pH}=6$ and under illumination with a $300 \mathrm{~W}$ xenon lamp. An $\mathrm{Ag} / \mathrm{AgCl}$ electrode in a saturated $\mathrm{KCl}$ solution was used as a reference electrode and a $\mathrm{Pt}$ wire served as a counter electrode. The $I-V$ measurement of the $\mathrm{ZnO}-\mathrm{ZnGa}_{2} \mathrm{O}_{4}$ photoanode at an applied potential of $0.7 \mathrm{~V}$ (versus $\mathrm{Ag} / \mathrm{AgCl}$ electrode) was performed for $15 \mathrm{~min}$. As shown in figure 8(b), a low current density of $<10^{-4} \mathrm{~mA} \mathrm{~cm}{ }^{-2}$ was obtained in the dark and a high and stable current of $1.2 \mathrm{~mA} \mathrm{~cm}{ }^{-2}$ was measured under illumination with the xenon lamp for $15 \mathrm{~min}$. The stable photocurrent observed under continuous light illumination revealed that the $\mathrm{ZnGa}_{2} \mathrm{O}_{4}$ shells worked as an anticorrosive outer-layer of the $\mathrm{ZnO} \mathrm{NWs}$, enhancing the stability of the photoanode in comparison to the $\mathrm{ZnO}$ or $\mathrm{n}$-doped $\mathrm{ZnO} \mathrm{NWs}$, whose PEC performances deteriorate under continuous light illumination [2].

\section{Conclusions}

In summary, an easily controlled one-step CVD approach has been developed for a large-scale fabrication of the dense $\mathrm{ZnO}$ $\mathrm{NW}$ array on a $\mathrm{ZnO}$ film structure on the $a$-plane sapphire substrate, assisted with Au acting as a catalyst. The XRD pole figure and XRD rocking curve analyses revealed that the $\mathrm{ZnO} \mathrm{NW}$ array on a $\mathrm{ZnO}$ film structure consists of a single crystal domain with the $\mathrm{ZnO} c$-axis aligned perpendicularly to the $a$-plane sapphire substrate. STEM-EDS mapping and TEM ED studies also confirm the formation of a single crystal $\mathrm{ZnO}$ domain and the existence of a spinel $\mathrm{ZnAl}_{2} \mathrm{O}_{4}$ 
(a)

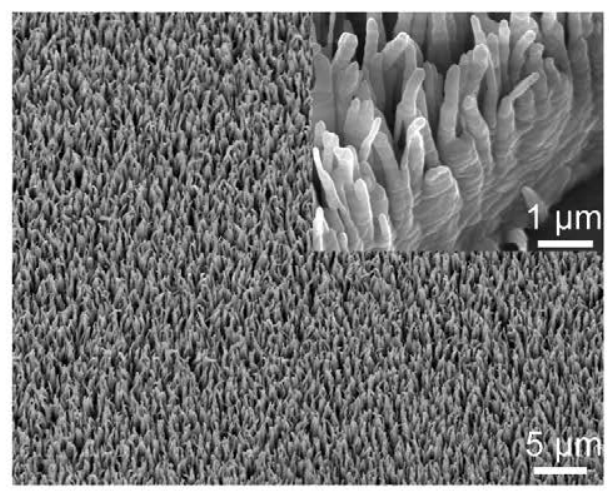

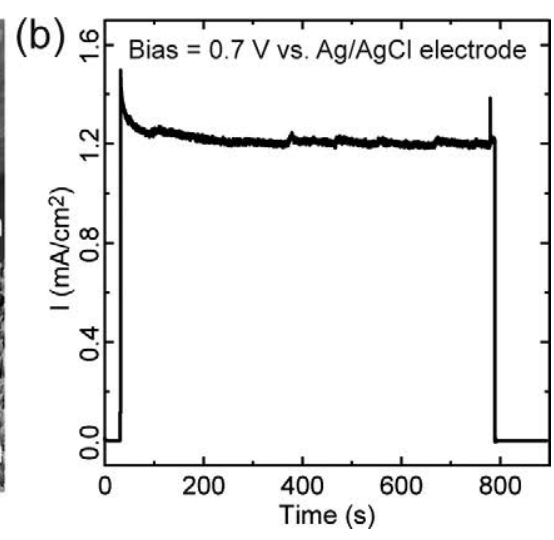

Figure 8. (a) Tilted-angle SEM image of a dense $\mathrm{ZnO}-\mathrm{ZnGa}_{2} \mathrm{O}_{4}$ core-shell nanowire array. Inset in (a) is a close-up of the vertically aligned $\mathrm{ZnO}-\mathrm{ZnGa}_{2} \mathrm{O}_{4}$ nanowires showing their roots bonded to the $\mathrm{ZnO}$ underlying film. (b) An $I-V$ curve of the $\mathrm{ZnO}-\mathrm{ZnGa}_{2} \mathrm{O}_{4}$ core-shell nanowire array on a $\mathrm{ZnO}$ film sample used as a photoanode at an applied bias of $0.7 \mathrm{~V}$ (versus $\mathrm{Ag} / \mathrm{AgCl}$ ) under illumination with a xenon lamp for about $15 \mathrm{~min}$.

intermediate layer with the $[0 \overline{2} 1]$ orientation between the epi-ZnO film and the $a$-plane sapphire substrate. The matched in-plane symmetry and an improved lattice matching between the spinel $\mathrm{ZnAl}_{2} \mathrm{O}_{4}(02 \overline{1})$ layer and the wurtzite $\mathrm{ZnO}(0001)$ film diminishes the possible strain present in the $\mathrm{ZnO}$ film and explains the high-quality epitaxial growth of the $\mathrm{ZnO}$ nanostructures. Both free and bound exciton emissions along with fine-featured phonon replica emissions are clearly observed in the low-temperature PL spectrum, indicating a good crystalline quality of the fabricated $\mathrm{ZnO}$ structure. The studied $\mathrm{ZnO}$ structures exhibit an n-type conductivity and a carrier density of $\sim 10^{17} \mathrm{~cm}^{-3}$, as determined by the electrochemical impedance analysis. Finally, we report the application of the $\mathrm{ZnO} \mathrm{NWs}$ on a $\mathrm{ZnO}$ film structure as a template for the fabrication of a single crystal $\mathrm{ZnO}-\mathrm{ZnGa}_{2} \mathrm{O}_{4}$ core-shell $\mathrm{NW}$ array. The $\mathrm{ZnO}-\mathrm{ZnGa}_{2} \mathrm{O}_{4}$ core-shell NW array on a film reveals an improved anticorrosive ability and an enhanced photoelectrochemical performance when used as a photoanode in a PEC water splitting cell. We expect the proposed $\mathrm{ZnO} \mathrm{NWs}$ fabrication technique to provide an easy and reliable approach for the large-scale fabrication of high-quality single crystalline domain $\mathrm{ZnO}$ nanostructures. We are convinced that such a straightforward fabrication technique will contribute efficiently to the integration of the $\mathrm{ZnO}$ nanostructures in devices with an enhanced light collection and light emission ability, such as photovoltaic cells, photoelectrochemical cells and light emitting diodes.

\section{Acknowledgments}

This work was supported through the Grant-in-Aid for Scientific Research (B) 22360056 from the Japan Society for the Promotion of Science (JSPS) and Ministry of Education, Culture, Sports, Science and Technology (MEXT), the Grant-in-Aid from the Asahi Glass Foundation, the Grant-in-Aid for JSPS Fellows and the Global COE program, 'Global Center of Excellence for Mechanical Systems Innovation' by MEXT. Part of this work was also conducted in the Research Hub for Advanced Nano Characterization, The University of Tokyo, supported by MEXT, Japan.

\section{References}

[1] Wang X, Song J, Summers C J, Ryou J H, Li P, Dupuis R D and Wang Z L 2009 J. Phys. Chem. B 1107720

[2] Yang X, Wolcott A, Wang G, Sobo A, Fitzmorris R C, Qian F, Zhang J Z and Li Y 2009 Nano Lett. 92331

[3] Zhang X X, Zhao D, Gao M, Dong H B, Zhou W Y and Xie S S 2011 Nanotechnology 22135603

[4] Fan H J et al 2006 Small 2561

[5] Yang P, Yan H, Mao S, Russo R, Johnson J, Saykally R, Morris N, Pham J, He R and Choi H 2002 Adv. Funct. Mater. 12323

[6] Zhong M, Li Y, Yamada I and Delaunay J-J 2012 Nanoscale 41509

[7] Vayssieres L 2003 Adv. Mater. 15464

[8] Tian Z R, Voigt J A, Liu J, Mckenzie B, Mcdermott M J, Rodriguez M A, Konishi H and Xu H 2003 Nature Mater. 2821

[9] Baxter J B and Aydil E S 2005 J. Cryst. Growth 274407

[10] Fons P, Iwata K, Yamada A, Matsubara K, Niki S, Nakahara K, Tanabe T and Takasu H 2000 Appl. Phys. Lett. 771801

[11] Volk J, Nagata T, Erdélyi R, Bársony I, Tóth A L, Lukács I E, Czigány Zs, Tomimoto H, Shingaya Y and Chikyow T 2009 Nanoscale Res. Lett. 4699

[12] Teo S H G, Liu A Q, Singh J, Yu M B and Lo G Q 2007 Appl. Phys. A 89417

[13] Fan H J, Lee W, Scholz R, Dadgar A, Krost A, Nielsch K and Zacharias M 2005 Nanotechnology 16913

[14] Park W I, Kim D H, Jung S W and Yi G 2002 Appl. Phys. Lett. 804232

[15] Chen Y, Bagnall D M, Koh H, Park K, Hiraga K, Zhu Z and Yao T 1998 J. Appl. Phys. 843912

[16] Levin I, Davydov A, Nikoobakht B, Sanford N and Mogilevsk P 2005 Appl. Phys. Lett. 87103110

[17] Engler O and Randle V 2010 Introduction to Texture Analysis: Macrotexture, Microtexture, and Orientation Mapping 2nd edn (Boca Raton, FL: CRC Press), (London: Taylor and Francis)

[18] Pennycook S J and Jesson D E 1990 Phys. Rev. Lett. 64938

[19] Yang Y, Kim D S, Knez M, Scholz R, Berger A, Pippel E, Hesse D, Gösele U and Zacharias M 2008 J. Phys. Chem. C 1124068

[20] Grabowska J, Kumar R R T, McGlynn E, Nanda K K, Newcomb S B, McNally P J, O'Reilly L, Mosnier J P and Henry M O 2008 Thin Solid Films $\mathbf{5 1 6} 1725$

[21] Wang Y, Liao Q, Lei H, Zhang X, Ai X, Zhang J and Wu K 2006 Adv. Mater. 18943 
[22] Polacer K and Wassermann E F 1976 Thin Solid Films 3765

[23] Wagner R S and Ellis W C 1965 Trans. Metall. Soc. AIME 489

[24] Hannon J B, Kodambaka S, Ross F M and Tromp R M 2006 Nature $\mathbf{4 4 0} 69$

[25] Kim D S, Scholz R, Gösele U and Zacharias M 2008 Small 41651

[26] Teke A, Özgür Ü, Doğan S, Gu X and Morkoc H 2004 Phys. Rev. B 70195207
[27] Meyer B K et al 2004 Phys. Status Solidi b 241231

[28] Wolcott A, Smith W A, Kuykendall T R, Zhao Y and Zhang J Z 2009 Adv. Funct. Mater. 191849

[29] Allen M W, Alkaisi M M and Durbin S M 2006 Appl. Phys. Lett. 89103520

[30] Mora-Seró I, Fabregat-Santiago F, Denier B and Bisquert J 2006 Appl. Phys. Lett. 89203117

[31] Zhong M, Li Y, Tokizono T, Yamada I and Delaunay J-J 2012 J. Nanopart. Res. 14804 